\title{
Modelado de las Relaciones Cuantitativas Estructura-Actividad (QSAR) de Análogos del Tipifarnib con Actividad Antichagásica
}

\author{
Kevin Granados-Tavera', Elkin A. Tilvez ${ }^{1}$ y Maicol Ahumedo-Monterrosa ${ }^{2}$ \\ (1) Programa de Química, Facultad de Ciencias Básicas. Universidad de la Amazonia, Florencia-180002, \\ Caquetá, Colombia \\ (2) CIPTEC, Programa de Tecnología en Producción Industrial, Fundación Universitaria Tecnológico \\ Comfenalco, Cartagena, Colombia (e-mail: e.tilvez@udla.edu.co)
}

Recibido Mar. 22, 2018; Aceptado May. 29, 2018; Versión final Jul. 30, 2018, Publicado Feb. 2019

\begin{abstract}
Resumen
En este trabajo aplicamos el procedimiento Relación Cuantitativa Estructura-Actividad (RCEA, o QSAR por sus siglas en inglés) a 33 compuestos derivados del Tipifarnib. Esto con el objetivo de identificar las características estructurales responsables de la actividad antichagásica contra el parasito del Tripanosomas cruzi, realizando una simulación con 33 análogos del Tipiofanib al nivel de teoría B3LYP/6-31+(d,p), seguido de la evaluación de 1632 descriptores en la plataforma E-Dragon online. Los descriptores RDF040v, E1p y R3e se utilizaron para construir los modelos, presentando buena calidad estadística. La validación presento valores de $R^{2}=0.89, Q^{2}=0.86$ y un $R^{2} m=0.47$. Las 18 moléculas que se proponen muestran un gran potencial farmacológico, particularmente, los compuestos $52,53,54$ y 55 que presentan un alto potencial de actividad $\mathrm{EC}_{50}$ con valores estimados de $0.33,0.18,0.18$, y $0.34 \mathrm{nM}$ respectivamente.
\end{abstract}

Palabras clave: RCEA; Tripanosomas cruzi; enfermedad de chagas; Tipifarnib; fármacos

\section{Modelling of the Structure-Activity Quantitative Relationships (QSAR) of Tipifarnib Analogues with Antichagasic Activity}

\begin{abstract}
The method known as Quantitative Structure-Activity Relationship (QSAR) was applied to 33 Tipifarnib derived compounds. This was done with the aim of identifying the structural features responsible for the antichagasic activity against the Trypanosoma cruzi parasite, by simulating 33 molecules at the level of theory B3LYP/6$31+(d, p)$, followed by the evaluation of 1632 descriptors on the E-Dragon online platform. The model constructed by the descriptors RDF040v, E1p and R3e proved to be robust and predictive. The cross-validation yielded good values $\left(R^{2}=0.89 ; Q^{2}=0.85\right)$, and $R^{2}=0.47$. The proposed 18 molecules show a great pharmacological potential, particularly the $52,53,54$ y 55 with an estimated $\mathrm{EC}_{50}$ of $0.33,0.18,0.18$, and 0.34 $\mathrm{nM}$ respectively.
\end{abstract}

Keywords: QSAR; Trypanosoma cruzi; chagas desease; Tipifarnib; drugs 


\section{INTRODUCCIÓN}

Durante las últimas décadas las regiones tropicales menos favorecidas fueron severamente afectadas por enfermedades infecciosas, estas enfermedades pueden prosperar en dichas regiones, debido a que los factores ambientales y biológicos les favorece, ya que pueden soportan altos niveles de biodiversidad de los agentes patogénicos y vectoriales. Otro aspecto fundamental es el factor social y político que socavan los esfuerzos para controlar estas infecciones (Valerio et al., 2002). Este tipo de padecimiento es conocido mundialmente como enfermedades tropicales olvidadas, las cuales perjudican a muchos países y afectan a miles de millones de personas. El mal de Chagas, es una enfermedad potencialmente mortal causada por el parásito protozoo Tripanosoma cruzi (T. cruzi), puede ser transmitida por virus como el dengue o bacterias como la Mycobacterium tuberculosis, parásitos como T. cruzi, insectos como Triatoma infestans, mediante transfusiones de sangre de la madre al feto, trasplantes de órganos o la ingestión oral de alimentos infestados (Rueda et al. ,2013). Se calcula que en el mundo hay entre 6 y 7 millones de personas infectadas por T. cruzi, el parásito causante de la enfermedad de Chagas, la mayoría de ellas en América Latina. (WHO, 2018)

Con respecto a las manifestaciones clínicas, existen dos etapas que caracterizan la enfermedad de Chagas. La primera etapa comienza justo después de la infección (fase aguda), y aunque es asintomática, se han reportado casos con síntomas similares a los de una infección febril, dificultando así su correcto diagnóstico (Rassi et al., 2010). La segunda etapa (fase crónica), que puede tardar hasta 15 años en aparecer, se caracteriza por cardiomiopatía dilatada y afecciones del tracto digestivo14 que representan un alto riesgo comprometiendo la vida de los pacientes (Gascón et al., 2007).

En cuanto al tratamiento de la enfermedad, se basa principalmente en dos fármacos nitro-heterocíclicos llamados Nifurtimox y benznidazol, descubiertos empíricamente entre 1960 y 1970, mostrando una efectividad en casi el $60 \%$ de los casos que son tratados durante la fase aguda. Sin embargo, el uso de estos dos medicamentos para el tratamiento de la enfermedad durante la frase crónica es controversial debido a que los nitro-heterociclos solo son eficaces contra la forma extracelular de $T$. cruzi que se desarrolla durante la fase aguda, además los fármacos presentan efectos secundarios en los pacientes y necesitan largos periodos de administración. Por lo tanto, la búsqueda de nuevos fármacos más eficaces contra la enfermedad de Chagas es necesaria

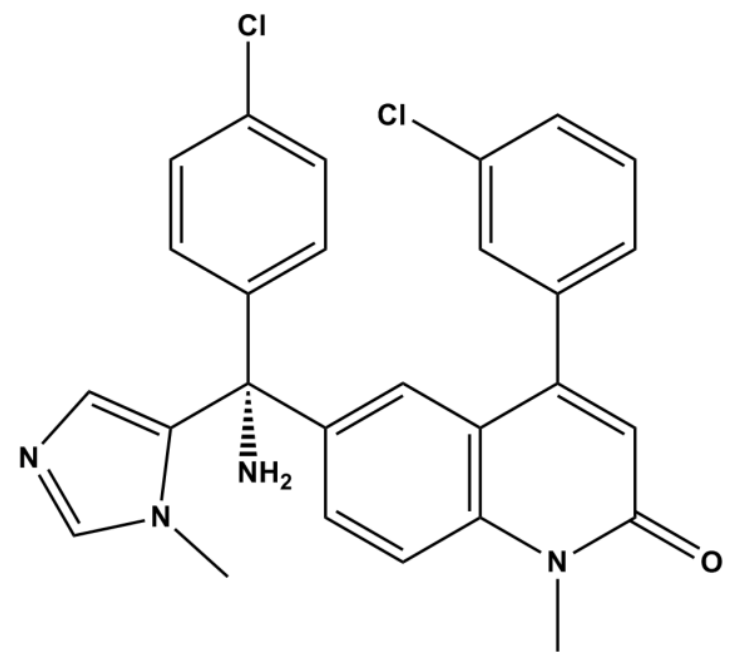

Fig. 1: Estructura química del Tipifarnib.

El Tipifarnib es un compuesto que tiene un alto grado de biodisponibilidad oral, tiene propiedades farmacocinéticas ideales, y es bien tolerado en humanos, además de tener buena actividad frente al parasito, por tanto, se considera que este compuesto tiene una ventaja excepcional para el desarrollo de fármacos contra la enfermedad de Chagas (James et al., 2010), este compuesto es un candidato a fármaco contra el cáncer desarrollado por Johnson and Johnson Pharmaceuticals (Karp et al., 2001), su estructura se muestra en la Figura 1. Experimentos demostraron que el mecanismo primario de acción del Tipifarnib en el parásito fue un bloqueo de la biosíntesis de esteroles a través de la inhibición de CYP51. La síntesis de esteroles es esencial para la supervivencia en todas las etapas del ciclo de vida del parásito y es altamente susceptible a los inhibidores de la biosíntesis de esteroles. Se han diseñado algunos análogos del Tipifarnib donde reducen al mínimo la actividad contra la feniltrasferasa, mientras mejoran la actividad inhibitoria contra CYP51. Los compuestos sintetizados demuestran actividad inhibitoria subnanomolar en el amastigotes intracelular de $T$. cruzi y actividad represiva altamente potente en la infección por T. cruzi en el modelo del ratón (Buckner et al., 2012). 
Debido al gran esfuerzo y costo que genera la implementación de métodos de síntesis de nuevos fármacos y ensayos de evaluación biológica de derivados de Tipifarnib, se necesitan estrategias que permitan optimizar la síntesis de nuevos compuestos con posible actividad biológica y así, poder disminuir los efectos secundarios de estos. La química computacional se ha convertido en una herramienta importante ya que nos permiten la creación de modelos capaces de complementar estudios experimentales, y además su ventaja principal es que son más económicos y controlables (Bajorath et al., 2015). En la presente investigación, se realizó un análisis de las relaciones cuantitativas estructura-actividad (QSAR por sus siglas en Inglés Quantitative structure-activity relationship) (Kubinyi, 2004) para determinar las propiedades moleculares relacionadas con la eficiencia de los compuestos reportados por James y colaboradores (James et al., 2010). El modelo QSAR obtenido, permite estimar la actividad de compuestos no ensayados experimentalmente, al igual que nuevos fármacos propuestos teóricamente. Estos modelos generados deben ser validados por diferentes métricas, siendo el objetivo principal de la validación emitir un juicio con un alto grado de confianza sobre los errores encontrados durante la predicción del conjunto de prueba dentro del dominio del modelo (López et al., 2014; Ensuncho et al., 2017).

Tabla 1: Análogos del Tipifarnib con modificaciones en los grupos Ar y Ar' contra el parásito T. cruzi. Los valores de IC $_{50}$ (James et al., 2010), son reportados en nM

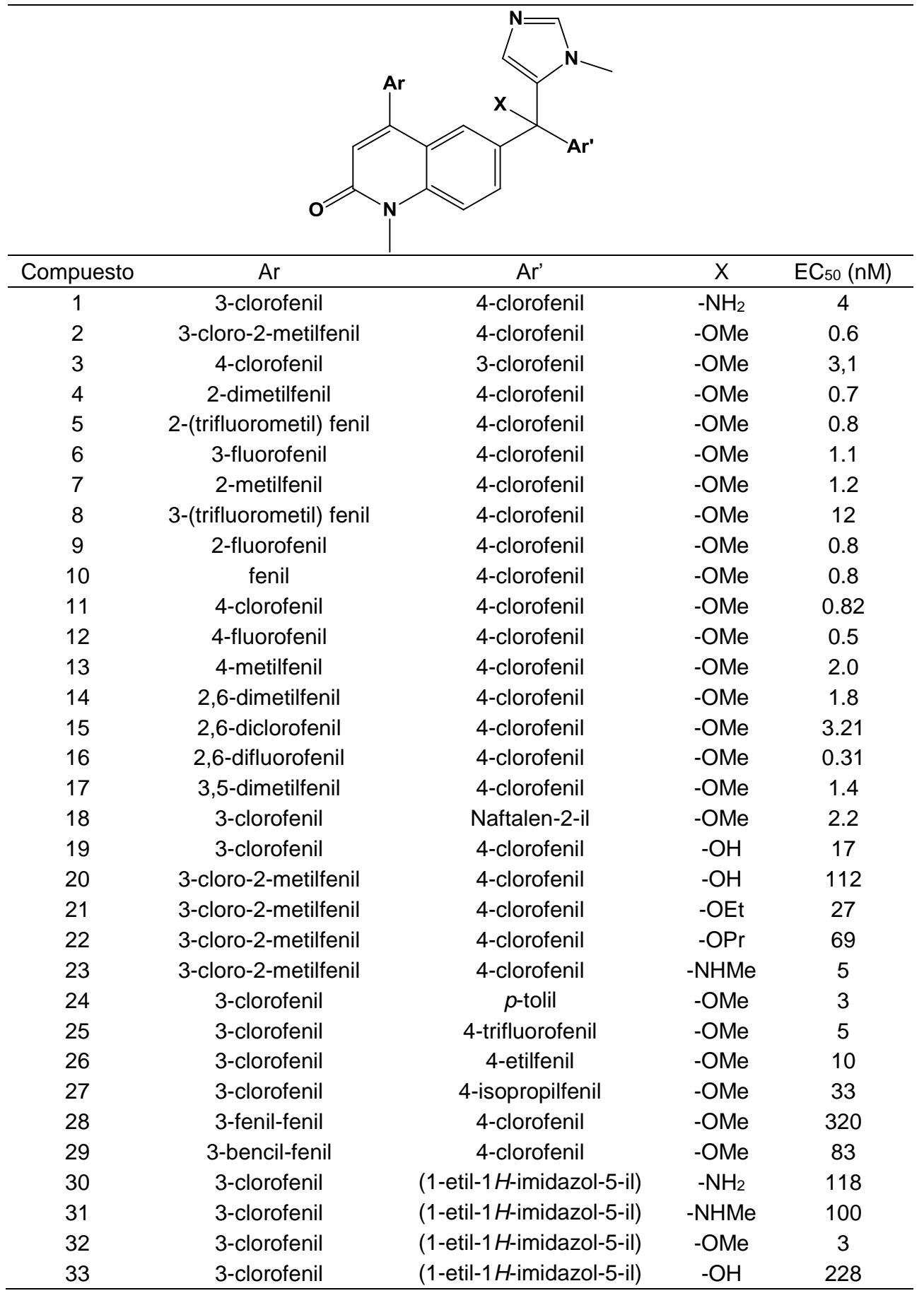




\section{METODOLOGÍA}

Una serie de 33 compuestos derivados de Tipifarnib reportados (Kraus et al.,.2010) con actividad $\mathrm{EC}_{50}$ contra $T$. cruzi, fueron tomados para este estudio. La estructural general de los compuestos y sus respectivas actividades reportadas con $\mathrm{EC}_{50}$ contra T.cruzi se muestra en la Tabla 1. Las geometrías de 33 moléculas objeto de estudio se optimizaron utilizando la Teoría del Funcional de la Densidad (DFT), al nivel de teoría B3LYP/6-31+(d,p), el cual ha sido reportado al generar buenas estructuras de partida para estudios de tipo QSAR (Nikolic et al., 2008). Las moléculas que no lograron converger a este nivel de teoría se le realizaron optimización al nivel de teoría HF/STO-3G para corregir problemas geométricos, una vez corregida la estructura optimizado al nivel B3LYP/6-31+(d,p).Todos los cálculos mencionados anteriormente se realizaron utilizando el paquete computacional ORCA (Neese, 2012).

La plataforma E-Dragon online fue utilizada para obtener los descriptores moleculares relacionados con el tipo de átomo, grupo funcional, conteos de fragmentos, descriptores topológicos y geométricos (Tetko et al., 2005). Posteriormente se realizó la selección de variables mediante el análisis de regresión lineal, utilizando el programa Excel. Los modelos QSAR se obtuvieron a través de un análisis de Regresión lineal múltiple y se utilizó el esquema de validación cruzada (La validación interna de los modelos construidos) "dejando uno afuera" (LOO, siglas en inglés para Leave one out). A partir el procedimiento de validación cruzada LOO, se obtiene el cuadrado del coeficiente de validación cruzada LOO $\left(\mathrm{Q}^{2}\right)$, que se utiliza como un criterio para evaluar tanto la robustez y la capacidad predictiva de los modelos generados, el resto de los parámetros estadísticos se calcularon y se analizaron para evaluar la calidad estadística de los modelos QSAR propuestos y así seleccionar el mejor de ellos.

Para estimar de forma confiable la capacidad predictiva de nuestro modelo QSAR se realizó una validación externa, donde se realiza la predicción de la variable dependiente de un conjunto de prueba externo de compuestos, que no se utilizó para la construcción del modelo. La validación externa es una de las etapas más importantes cuando se desarrollan modelos QSAR, ya que esta confirma la robustez y la capacidad predictiva del modelo (Veerasamy et al., 2011). De manera que se generaron dieciocho nuevas moléculas a partir de la modificación en los grupos Ar y Ar' de la molécula del Tipifarnib, así como la variación del grupo $-\mathrm{NH}_{2}$ por $-\mathrm{OMe}$, con el objeto de predecir la actividad antiparasitaria utilizando los modelos propuestos

\section{RESULTADOS}

Se calcularon 1632 descriptores moleculares en la plataforma E-Dragon online. Para seleccionar los descriptores que harían parte del modelo QSAR se realizó un análisis de regresión lineal para cada descriptor, con el fin de elegir los descriptores predominantes que explican la actividad inhibitoria de estos compuestos análogos a Tipifarnib. Los descriptores que presentaron un coeficiente de determinación $\left(\mathrm{R}^{2}\right)$ cercano o mayor a 0.5 fueron seleccionados para la creación del modelo (Scior et al., 2009), en total se seleccionaron 8 descriptores en términos del cuadrado de los coeficientes de correlación $\left(R^{2}\right)$ (ver Tabla 2). Si existe correlación entre las variables independiente de un modelo QSAR resulta difícil saber si los cambios en la actividad biológica son debido a un descriptor en específico, por lo tanto, es necesario analizar el grado de correlación entre los descriptores (ver Tabla 3).

Los mayores grados de correlación se producen entre descriptores de la misma naturaleza, en consecuencia, es apropiado seleccionar descriptores de diferente naturaleza para garantizar la robustez del modelo. Las ecuaciones 1 y 2 corresponden a los modelos QSAR obtenidos (M1 y M2) utilizando los datos mostrados en la Tabla 1 como conjunto de entrenamiento, la actividad biológica se expresó como -Log (E- $\left.\mathrm{C}_{50}\right)$ dada su conveniencia en el análisis de regresión lineal.

M1: $\quad-\log \left(E_{50}\right)=-0.2259(R D F 040 v)+7.7574(E 1 p)-4.2605(R 3 e)+14.3164$

M2: $\quad-\log \left(E C_{50}\right)=-0.1795(R D F 040 p)+6.6988(E 1 u)-4.6633(R 3 e)+15.0524$

La Tabla 4 muestra los parámetros estadísticos obtenidos para los modelos $M 1$ y M2. $R^{2}$ es el coeficiente de regresión múltiple, $\mathrm{F}$ es la prueba de Fischer, $\mathrm{Q}^{2}$ es el coeficiente de correlación de la validación cruzada (LOO-CV), PRESS (predicted residual sums of squares) es la suma de los cuadrados de error de predicción, SSY (sum of squares of $Y$ ) es la suma de cuadrados correspondiente a la variable dependiente y la expresión PRESS/SSY es el intervalo de confianza aproximado para una predicción. Un modelo de regresión es aceptable si $R^{2}>0.6$, también se requiere que el valor del error estándar sea próximo a cero debido a que esto implica mayor confianza en la predicción (Guerra et al., 2016). El error de la validación debe ser cercano a cero, el valor PRESS debe ser menor a SSY y la expresión PRESS/SSY debe ser menor a 0.4 y si el valor es 0.1 quiere decir que el modelo significativamente aceptable. Todos estos es para demostrar que los 
modelos desarrollados tienen buena predicción y puede considerarse estadísticamente significativos (Can, 2014). Los modelos anteriormente mencionados (Ecuaciones 1 y 2) cumplen con dichos parámetros estadísticos

Tabla 2: Actividad biológica expresada como (-Log $E_{50}$ ) y valores de los descriptores moleculares empleados en los modelos propuestos.

\begin{tabular}{|c|c|c|c|c|c|c|c|c|c|}
\hline Compuesto & $\begin{array}{c}\text { Actividad } \\
\text { Experimental }\end{array}$ & RDF040v & $R D F 040 p$ & E1u & E1v & E1e & E1p & R3u & R3e \\
\hline 1 & 8.40 & 13.223 & 15.607 & 0.549 & 0.461 & 0.562 & 0.467 & 1.622 & 1.655 \\
\hline 2 & 9.22 & 13.558 & 16.159 & 0.598 & 0.536 & 0.612 & 0.553 & 1.557 & 1.599 \\
\hline 3 & 8.51 & 14.702 & 16.949 & 0.616 & 0.58 & 0.632 & 0.603 & 1.544 & 1.591 \\
\hline 4 & 9.15 & 11.56 & 13.684 & 0.602 & 0.534 & 0.615 & 0.548 & 1.57 & 1.586 \\
\hline 5 & 9.10 & 11.052 & 12.764 & 0.612 & 0.544 & 0.629 & 0.56 & 1.556 & 1.617 \\
\hline 6 & 8.96 & 15.169 & 17.313 & 0.603 & 0.559 & 0.622 & 0.572 & 1.567 & 1.63 \\
\hline 7 & 8.92 & 11.493 & 13.432 & 0.588 & 0.538 & 0.604 & 0.552 & 1.554 & 1.574 \\
\hline 8 & 7.92 & 11.549 & 13.398 & 0.489 & 0.444 & 0.528 & 0.447 & 1.523 & 1.599 \\
\hline 9 & 9.10 & 10.894 & 12.574 & 0.601 & 0.547 & 0.611 & 0.563 & 1.56 & 1.605 \\
\hline 10 & 9.10 & 11.277 & 13.217 & 0.584 & 0.52 & 0.599 & 0.533 & 1.557 & 1.575 \\
\hline 11 & 9.09 & 12.852 & 15.2 & 0.584 & 0.518 & 0.598 & 0.531 & 1.539 & 1.59 \\
\hline 12 & 9.30 & 11.129 & 13.027 & 0.584 & 0.521 & 0.597 & 0.534 & 1.55 & 1.623 \\
\hline 13 & 8.70 & 11.353 & 13.311 & 0.51 & 0.486 & 0.533 & 0.494 & 1.527 & 1.55 \\
\hline 14 & 8.74 & 14.021 & 16.606 & 0.601 & 0.53 & 0.614 & 0.542 & 1.562 & 1.577 \\
\hline 15 & 8.49 & 15.596 & 18.72 & 0.593 & 0.542 & 0.608 & 0.557 & 1.55 & 1.607 \\
\hline 16 & 9.51 & 10.322 & 11.906 & 0.599 & 0.547 & 0.613 & 0.561 & 1.558 & 1.641 \\
\hline 17 & 8.85 & 11.976 & 14.031 & 0.518 & 0.481 & 0.527 & 0.492 & 1.516 & 1.537 \\
\hline 18 & 8.66 & 12.281 & 14.418 & 0.514 & 0.49 & 0.529 & 0.504 & 1.645 & 1.654 \\
\hline 19 & 7.77 & 13.371 & 15.401 & 0.536 & 0.471 & 0.55 & 0.486 & 1.63 & 1.682 \\
\hline 20 & 6.95 & 14.677 & 17.014 & 0.512 & 0.483 & 0.532 & 0.496 & 1.648 & 1.693 \\
\hline 21 & 7.57 & 15.66 & 18.613 & 0.569 & 0.504 & 0.578 & 0.524 & 1.596 & 1.632 \\
\hline 22 & 7.16 & 15.056 & 17.945 & 0.5 & 0.427 & 0.501 & 0.447 & 1.634 & 1.654 \\
\hline 23 & 8.30 & 15.699 & 18.545 & 0.576 & 0.496 & 0.581 & 0.514 & 1.598 & 1.625 \\
\hline 24 & 8.52 & 12.452 & 14.498 & 0.494 & 0.464 & 0.511 & 0.476 & 1.566 & 1.575 \\
\hline 25 & 8.30 & 12.27 & 14.242 & 0.49 & 0.465 & 0.532 & 0.468 & 1.558 & 1.627 \\
\hline 26 & 8.00 & 12.595 & 14.698 & 0.434 & 0.397 & 0.44 & 0.413 & 1.663 & 1.656 \\
\hline 27 & 7.48 & 12.889 & 15.105 & 0.456 & 0.416 & 0.458 & 0.434 & 1.715 & 1.709 \\
\hline 28 & 6.49 & 16.878 & 19.724 & 0.438 & 0.413 & 0.429 & 0.431 & 1.704 & 1.703 \\
\hline 29 & 7.08 & 13.95 & 16.691 & 0.407 & 0.361 & 0.396 & 0.375 & 1.743 & 1.74 \\
\hline 30 & 6.93 & 15.732 & 18.431 & 0.47 & 0.474 & 0.495 & 0.492 & 1.723 & 1.752 \\
\hline 31 & 7.00 & 15.986 & 19.051 & 0.519 & 0.445 & 0.528 & 0.462 & 1.627 & 1.655 \\
\hline 32 & 8.52 & 13.887 & 16.312 & 0.56 & 0.523 & 0.58 & 0.54 & 1.589 & 1.627 \\
\hline 33 & 6.64 & 15.197 & 17.293 & 0.523 & 0.428 & 0.529 & 0.442 & 1.664 & 1.705 \\
\hline
\end{tabular}

Para los dos modelos se requiere de 3 variables independientes para predecir la actividad biológica; para M1 el coeficiente de correlación indica que la variabilidad de la actividad biológica es explicada en un $86.44 \%$ mientras que para M2 en un $84.31 \%$ por lo cual los dos modelos son aceptables.

Tabla 3: Matriz de correlación de los descriptores moleculares calculados

\begin{tabular}{ccccccccc}
\hline Descriptores & RDF040v & RDF040p & E1u & E1v & E1e & E1p & R3u & R3e \\
\hline RDF040v & 1 & & & & & & & \\
RDF040p & 0.995 & 1 & & & & & & \\
E1u & -0.214 & -0.216 & 1 & & & & & \\
E1v & -0.250 & -0.258 & 0.930 & 1 & & & & \\
E1e & -0.260 & -0.265 & 0.986 & 0.946 & 1 & & & \\
E1p & -0.208 & -0.215 & 0.928 & 0.997 & 0.935 & 1 & & \\
R3u & 0.494 & 0.488 & -0.700 & -0.712 & -0.754 & -0.673 & 1 & \\
R3e & 0.487 & 0.466 & -0.580 & -0.594 & -0.609 & -0.563 & 0.922 & 1 \\
\hline
\end{tabular}


El modelo M1 utiliza el descriptor RDF040v (radial distribution function - 4.0 / weighted by atomic van der Waals volumes), que forma parte de los descriptores de función de distribución radial, donde $\mathrm{R}$ es la distancia que puede tener valores de 1.0 a $15.5 \AA$, por consiguiente, las parejas con valores cercanos a $R$ juegan un rol importante en el descriptor, además proporciona información sobre longitudes de enlace, tipos de anillos, sistemas planos y no planos, tipos de átomos y peso molecular (Li et al., 2008). Para el descriptor, RDF040v, el coeficiente $R$ presenta un valor de $4.0 \AA$ y la sigla $v$ indica que está ponderado por el volumen atómico de van der Waals, lo cual indica que las distancias geométricas dentro de la estructura con valores de $4.0 \AA$, puede indicar la unión favorable de los derivados de Tipifarnib a la enzima diana CYP51. El descriptor E1p forma parte de los descriptores conocidos como WHIM (weighted holistic invariant molecular descriptors), el cual, es un índice molecular 3D que representan diferentes fuentes de información química en términos de tamaño, forma, simetría y distribución atómica y son obtenidos a partir de sus coordenadas de la estructura tridimensional, que al estar ponderado por la polarizabilidad atómica, puede también brindar información del molecular del estado electrotopológico.

Tabla 4: Valores estadísticos obtenidos para los modelos propuestos M1 y M2

\begin{tabular}{lcc}
\hline Resultados Estadísticos & $M 1$ & $M 2$ \\
\hline $\mathrm{n}$ & 33 & 33 \\
$\mathrm{R}^{2}$ & 0.8644 & 0.8431 \\
$\mathrm{~F}$ & 61.6228 & 51.9297 \\
$\mathrm{Q}^{2}$ & 0.8259 & 0.7192 \\
Error estándar & 0.3364 & 0.3619 \\
error de la validación & 0.1283 & 0.2118 \\
PRESS & 3.2823 & 3.7988 \\
SSY & 24.2062 & 24.2062 \\
PRESS/SSY & 0.1356 & 0.1569 \\
\hline
\end{tabular}

Por último, R3e ( $R$ autocorrelation of lag 3 / weighted by atomic Sanderson electronegativities), es un descriptor GETAWAY (geometric, Topology, and atoms weight assem/Y), el cual se relacionan con la influencia de los átomos en la determinación de la forma molecular. El descriptor R3e involucra átomos con una distancia topológica de 3 enlaces, promediados por la electronegatividad. Para M2, el descriptor E1u (1st component accessibility directional WHIM index / unweighted) es un descriptor geométrico basado en índices estadísticos calculados sobre las proyecciones de los átomos a lo largo de los ejes principales, se relaciona con el tamaño molecular, la forma y la simetría (Xinliang et al., 2012). Además, también se utilizan los descriptores R3e y RDF040p los cuales ya han sido ampliamente usados en otros estudios (Zhou et al., 2012).

Tabla 5: Valores obtenidos para los modelos propuestos M1 y M2 utilizando la técnica Jackknife.

\begin{tabular}{|c|c|c|c|c|c|c|c|}
\hline \multicolumn{2}{|c|}{ M1 } & \multicolumn{2}{|c|}{ M2 } & \multicolumn{2}{|c|}{ M1 } & \multicolumn{2}{|c|}{ M2 } \\
\hline Molécula & $R^{2}$ & Molécula & $R^{2}$ & Molécula & $R^{2}$ & Molécula & $R^{2}$ \\
\hline 1 & 0.875 & 1 & 0.844 & 18 & 0.866 & 18 & 0.851 \\
\hline 2 & 0.870 & 2 & 0.849 & 19 & 0.864 & 19 & 0.845 \\
\hline 3 & 0.872 & 3 & 0.845 & 20 & 0.877 & 20 & 0.847 \\
\hline 4 & 0.860 & 4 & 0.838 & 21 & 0.866 & 21 & 0.845 \\
\hline 5 & 0.862 & 5 & 0.862 & 22 & 0.859 & 22 & 0.839 \\
\hline 6 & 0.878 & 6 & 0.855 & 23 & 0.874 & 23 & 0.847 \\
\hline 7 & 0.868 & 7 & 0.845 & 24 & 0.864 & 24 & 0.843 \\
\hline 8 & 0.873 & 8 & 0.856 & 25 & 0.865 & 25 & 0.844 \\
\hline 9 & 0.864 & 9 & 0.841 & 26 & 0.871 & 26 & 0.851 \\
\hline 10 & 0.861 & 10 & 0.839 & 27 & 0.861 & 27 & 0.839 \\
\hline 11 & 0.865 & 11 & 0.841 & 28 & 0.844 & 28 & 0.819 \\
\hline 12 & 0.861 & 12 & 0.838 & 29 & 0.866 & 29 & 0.843 \\
\hline 13 & 0.867 & 13 & 0.843 & 30 & 0.855 & 30 & 0.833 \\
\hline 14 & 0.864 & 14 & 0.841 & 31 & 0.857 & 31 & 0.839 \\
\hline 15 & 0.867 & 15 & 0.848 & 32 & 0.864 & 32 & 0.845 \\
\hline 16 & 0.856 & 16 & 0.835 & 33 & 0.856 & 33 & 0.862 \\
\hline 17 & 0.862 & 17 & 0.841 & & & & \\
\hline
\end{tabular}




\section{Validación interna}

La validación de los modelos QSAR es de gran importancia ya que de esta forma se garantiza su habilidad predictiva. En general M1 y M2 presentan buen poder de predicción interna debido a que los coeficientes de correlación de la validación cruzada dejando uno afuera (LOO-CV) son superiores a 0.5 (Guerra et al., 2016). Si bien los modelos M1 y M2 son estadísticamente aceptables, las ecuaciones QSAR pueden mejorarse y obtener una mayor calidad estadística, de tal forma que se implementó la técnica Jackknife, en la cual un compuesto es considerado como valor atípico (outlier), si el valor de $\mathrm{R}^{2}$ que se obtiene a partir del análisis de regresión después de eliminar dicho compuesto, es comparativamente más alto que otros valores de $\mathrm{R}^{2}$ (Ensuncho et al., 2017).

De la Tabla 5, se puede observar que para M1 las estructuras 1, 6 y 20 presentan los mayores valores de $\mathrm{R}^{2}$ por lo que son considerados outliers. Para M2, los compuestos 5, 8 y 33 presentan los valores más altos de $\mathrm{R}^{2}$ por lo que al ser excluidos de los modelos mejora su calidad y significancia estadística. Atendiendo dichas observaciones, las ecuaciones 3 y 4 corresponden a los modelos obtenidos luego de aplicar la técnica jackknife, los cuales se designaron como M3 y M4 respectivamente.

$$
\begin{aligned}
& \text { M3: }-\log \left(E C_{50}\right)=-0.2339(R D F 040 v)+7.6015(E 1 p)-3.8613(R 3 e)+13.8379 \\
& M 4:-\log \left(E C_{50}\right)=-0.1926(R D F 040 p)+0.8025(E 1 u)-3.8345(R 3 e)+13.9044
\end{aligned}
$$

Tabla 6: Datos estadísticos obtenidos para los modelos M3 y M4, después de aplicar la técnica Jackknife

\begin{tabular}{lcc}
\hline Resultados Estadísticos & $M 3$ & $M 4$ \\
\hline $\mathrm{n}$ & 30 & 30 \\
$\mathrm{R}^{2}$ & 0.8994 & 0.8753 \\
$\mathrm{~F}$ & 77.4553 & 60.8352 \\
$\mathrm{Q}^{2}$ & 0.8654 & 0.8424 \\
Error estándar & 0.2917 & 0.3154 \\
error de la validación & 0.0991 & 0.1094 \\
$\mathrm{PRESS}$ & 2.2121 & 2.5864 \\
$\mathrm{SSY}$ & 21.9818 & 20.7413 \\
PRESS/SSY & 0.1006 & 0.1247 \\
\hline
\end{tabular}

Como se observa en las ecuaciones 3 y 4 , la calidad estadística de los modelos aumento, en particular, los valores de $R^{2}$ y $Q^{2}$ fueron más alto, el error estándar, el error de la validación, PRESS, SSY y PRESS/SSY reportaron valores menores respecto a los modelos M1 y M2. Adicionalmente el valor de F para los modelos M3 y M4 excede en mayor proporción el valor tabulado de $\mathrm{F}_{\mathrm{t}}=7.562(\alpha=0.01$; grados de libertad 1 y 30$)$ lo que indica una mayor significancia estadística en comparación con M1 y M2 (Guerra et al., 2016). Estos resultados demuestran que la exclusión de los compuestos en cada caso del análisis de regresión es determinante en la calidad estadística y la capacidad predictiva de los modelos QSAR reportados. En comparación, $\mathrm{M} 3$ es el mejor modelo ya que los valores $\mathrm{R}^{2}, \mathrm{Q}^{2}$ y $\mathrm{F}$ son mayores mientras que PRESS, SSY, PRESS/SSY, el error estándar y el error de la validación son menores respecto a M4. Cabe destacar que la expresión PRESS/SSY de M3 es cercano a 0.1 por lo cual, según lo mencionado anteriormente, el modelo es significativamente aceptable. La validación interna es un buen criterio para evaluar el poder predictivo de un modelo, pero no absoluto. Aunque, el bajo valor de $\mathrm{Q}^{2}$ para el conjunto de entrenamiento puede servir como un indicador de una baja capacidad de predicción de un modelo, lo contrario no es necesariamente cierto. De hecho, un alto valor $\mathrm{Q}^{2}$ no implica automáticamente una alta capacidad de predicción del modelo. Por este motivo, existe la necesidad de medidas de validación externa más rigurosas basadas en moléculas que no se utilizaron para el desarrollo del modelo (Tropsha.et al., 2003)

\section{Validación externa}

Para desarrollar el modelo y validarlo, se necesita dividir todo el conjunto de datos disponible en el conjunto de entrenamiento y prueba. En nuestro caso, para llevar a cabo la validación externa del modelo M3, se tomó como conjunto de entrenamiento a 30 moléculas $(2,3,4,5,7,8,9,10,11,12,13,14,15,6,17,18,19,21$, $22,23,24,25,26,27,28,29,30,31,32$ y 33) de las 33 estudiadas por James y colaboradores (James et al., 2010). Para la conformación del conjunto de prueba se seleccionaron las moléculas 1, 6, 20 (James et al., 2010), 34, 35, 36, (Kraus et al; 2009) y la molécula 37 que fue reportada Li et al., 2005, para un total de 7 
moléculas. En la Tabla 7 podemos comparar los resultados de las predicciones del conjunto de prueba y en la Tabla 8 se muestran las estructuras y los valores de $\mathrm{EC}_{50}$ de las 4 moléculas externas utilizadas en el conjunto de prueba.

Tabla 7: Residuos entre los valores experimentales y predichos (-Log $\mathrm{EC}_{50}$ ) para el conjunto de prueba.

\begin{tabular}{cccc}
\hline Molécula & $Y_{\text {EXP }}$ & $Y_{\text {PRED }}$ & $Y_{\text {RES }}$ \\
\hline 1 & 8.39 & 7.90 & 0.494 \\
6 & 8.95 & 8.34 & 0.62 \\
20 & 6.95 & 7.64 & -0.69 \\
34 & 7.64 & 7.20 & -0.44 \\
35 & 7.64 & 7.37 & -0.27 \\
36 & 7.92 & 7.87 & -0.05 \\
37 & 7.66 & 7.65 & -0.01 \\
\hline
\end{tabular}

Los resultados señalan un coeficiente de correlación de Pearson de 0.74 entre los valores predichos (Y PRED) y experimentales (YEXP) lo que indica una buena correlación lineal entre las variables (Alencar et al., 2016). Mientras el coeficiente de correlación $\mathrm{R}^{2}$ es 0.55 (ver Figura 2).

Tabla 8: Moléculas empleadas para la validación externa. Los valores de EC50 son medidos en nM

Compuesto




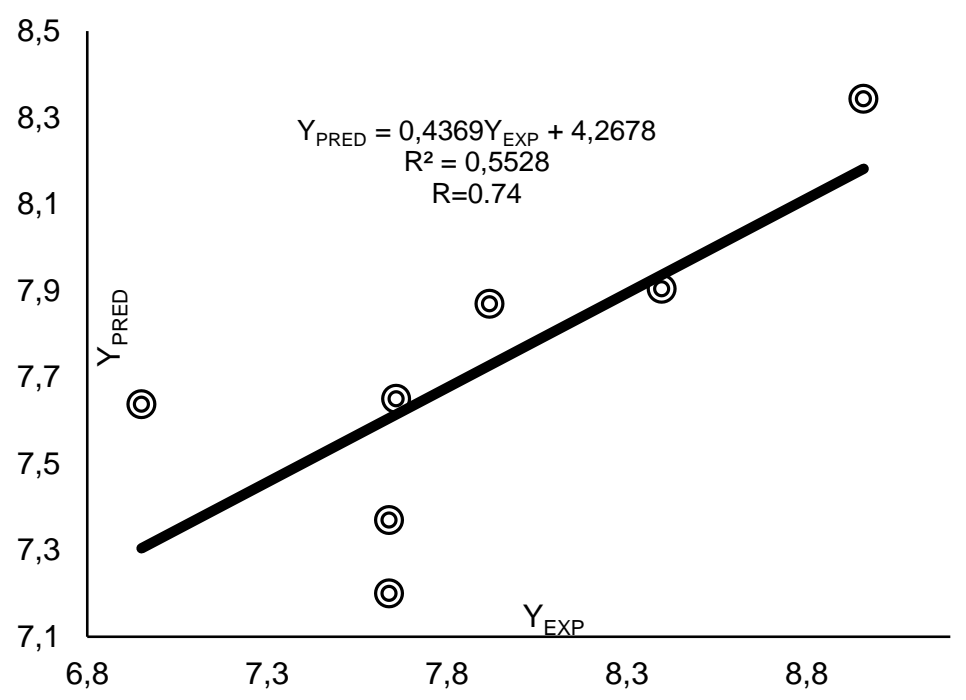

Fig. 2: Representación gráfica de la correlación entre los valores experimentales (YEXP) y predichos (YPRED) para el conjunto de prueba.

Un modelo QSAR se considera predictivo si las siguientes condiciones se cumplen (Tropsha, 2010): $\mathrm{Q}^{2}>0.5$; $R^{2}>0.6 ;\left(R^{2}-R^{2} 0\right) / R^{2}<0.1$ ó $\left(R^{2}-R^{\prime 2} 0\right) / R^{2}<0.1 ; 0.85<k<1.15$ ó $0.85<k^{\prime}<1.15$. Donde $R^{2}$ es el cuadrado del coeficiente de correlación entre las actividades experimentales y calculadas del conjunto de prueba. $R^{2}{ }_{0}$ y $\mathrm{R}^{\prime 2} \mathrm{~m}$ son coeficientes cuadrados de correlación del conjunto de prueba para regresiones a través del origen obtenidos a partir de actividades experimentales vs calculadas y calculadas vs experimentales, respectivamente. Por último, $\mathrm{k}$ y k' son las pendientes de las líneas de regresión a través del origen obtenidos a partir de actividades experimentales vs calculadas y calculadas vs experimentales, respectivamente. Adicional a estos parámetros estadísticos, se calculó la métrica $R^{2} \mathrm{~m}$, la cual determina la proximidad entre la actividad observada y la predicha (Roy et al., 2013; Roy et al., 2014). Un valor $\mathrm{R}^{2} \mathrm{~m}$ de mayor que 0.5 para un modelo QSAR indica que este tiene buena capacidad predictiva (ver Tabla 9).

Tabla 9: Parámetros de la validación externa para el modelo M3.

\begin{tabular}{cc}
\hline $\begin{array}{c}\text { Parámetros } \\
\text { Estadísticos }\end{array}$ & Modelo 3 \\
\hline $\mathrm{Q}^{2}$ & 0.86 \\
$\mathrm{R}^{2}$ & 0.55 \\
$\mathrm{R}^{2}{ }_{0}$ & 0.53 \\
$\mathrm{R}^{\prime 2}{ }_{0}$ & 0.99 \\
$\left(\mathrm{R}^{2}-\mathrm{R}^{2}{ }^{0}\right) / \mathrm{R}^{2}$ & 0.037 \\
$\left(\mathrm{R}^{2}-\mathrm{R}^{\prime}{ }^{2}\right) / \mathrm{R}^{2}$ & -0.8 \\
$\mathrm{k}$ & 1.02 \\
$\mathrm{k}$ & 0.97 \\
$\mathrm{R}^{2} \mathrm{~m}$ & 0.47 \\
\hline
\end{tabular}

De acuerdo con los parámetros estadísticos mostrados y teniendo en cuenta que cuatro de las siete moléculas utilizadas en el conjunto de prueba son externas a las 33 seleccionadas como moléculas de estudio, podemos establecer que el modelo QSAR construido cumple con los requisitos mínimos de calidad estadística para predecir la actividad biológica de nuevos análogos estructurales de Tipifarnib

\section{Diseño de nuevos compuestos}

Una vez verificado la robustez y calidad predictiva del modelo QSAR propuesto a través de la validación externa. Se pasó a seleccionar el mejor modelo propuesto, M3 (ver Ecuación 3) para poder estimar los valores de la actividad biológica del parasito $T$. cruzi de un conjunto de moléculas derivados del Tipifarnib, las cuales han sido propuestas teóricamente, conservando el esqueleto estructural del Tipifarnib. 
La Tabla 10 muestra las 18 moléculas propuestas con sus respectivas actividades estimadas con el modelo M3. Las principales modificaciones realizadas a la estructura del Tipifarnib, radicaron en el grupo Ar', donde las modificaciones se realizaron en la posición para- debido a que estudios comprobaron que en esta posición las moléculas presentan mayor actividad. (Guerra et al., 2016). Además, solo se plantearon estructuras con $\mathrm{Cl}, \mathrm{F}$ y $\mathrm{Br}$ debido a que en el estudio de referencia se encontró que las estructuras con $\mathrm{Cl}$ y $\mathrm{F}$ eran las moléculas que presentaban mayor actividad. Comparando estos 3 halógenos, se puede decir que las moléculas que tienen bromo en el grupo Ar' son las que presentan mayor actividad seguido de las moléculas con flúor y cloro. Es decir que el bromo y el flúor son los elementos que más contribuyen a la actividad de la molécula en comparación con el cloro. Con respecto al grupo Ar, se plantearon moléculas con diferentes halógenos en distintas posiciones, además de la presencia de otros grupos químicos en algunas moléculas Las moléculas que presentaron mayor actividad fueron las estructuras $52,53,54$ y 55 que tienen algo en común y es que tanto el grupo Ar y Ar' están constituidos por átomos de flúor y bromo, respetivamente, es decir que estos elementos son muy importantes en estas estructuras para aumentar la actividad antiparasitaria.

Tabla 10: Valores $\mathrm{EC}_{50}$ de lo derivados de Tipifarnib propuesto con el modelo M3.

Los valores de $\mathrm{EC}_{50}$, son medidos en $\mathrm{nM}$

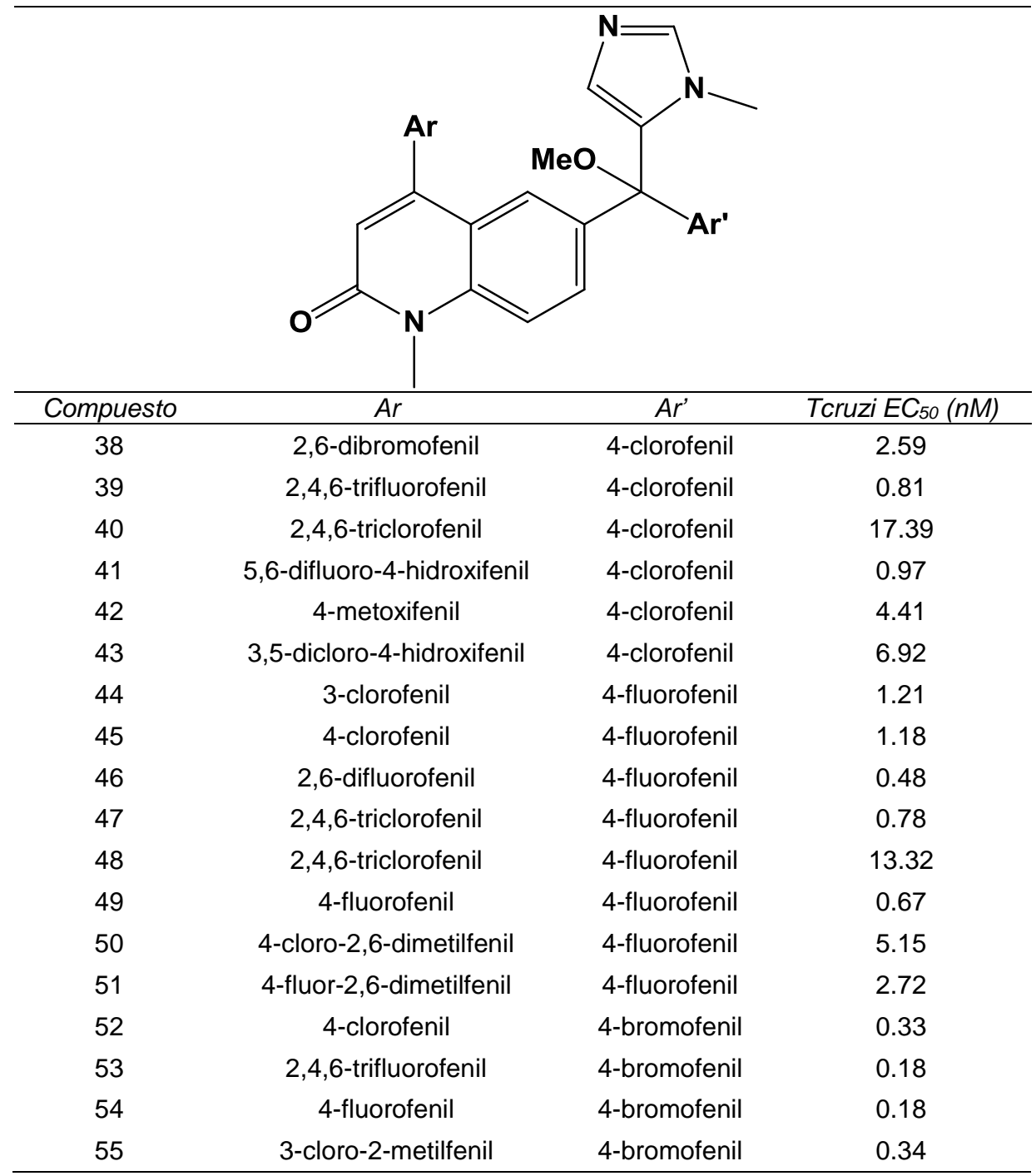

\section{CONCLUSIONES}

Se evaluaron un conjunto de 1632 descriptores moleculares en regresiones lineales múltiples con el fin evaluar la actividad antiparasitaria de compuestos derivados de Tipifarnib. Se logró establecer un modelo QSAR (M3) que relaciona los requerimientos estructurales y la actividad de moléculas derivadas del Tipifarnib frente al $T$. cruzi, los resultados sugieren que los descriptores RDF040v, E1p y R3e proporcionan información relacionada con la electronegatividad de los átomos y sus posiciones relativas para explicar la actividad de los compuestos estudiados. 
La validación interna señala que el modelo M3 cumple con los criterios mínimos de calidad estadística establecida en la literatura científica, además de tener una buena capacidad predictiva $\left(R^{2}=0.89\right.$ y $\left.Q^{2}=0.86\right)$. La validación externa del modelo M3 mostró criterios estadísticos $\left(R^{2}=0.55, R^{2} m=0.47\right)$, cercanos a los de referencia que son establecidos en los modelos con un alto poder predictivo, por lo tanto, puede ser considerado como un modelo valido para predecir las actividades de análogos estructurales de Tipifarnib.

Los nuevos derivados del Tipifarnib mostraron en su mayoría un gran potencial farmacológico, particularmente, los compuestos $52,53,54$ y 55, que presentan grupos con menor electronegatividad y estructuras moleculares alargadas, estas características pueden relacionarse con el patrón de sustitución en los anillos Ar y Ar', con base en ellos es posible diseñar otros compuestos potencialmente más activos.

\section{AGRADECIMIENTOS}

Los autores agradecen a la Vicerrectoría de Investigaciones y Posgrados de la Universidad de la Amazonia, por el soporte brindado durante la ejecución del proyecto de investigación código 600.6.84.

\section{REFERENCIAS}

Alencar, E.B. J.W. Castro y S.C.H. Cavalcanti, Quantitative Structure-Toxicity Relationships and Molecular Highlights about Aedes aegypti Larvicidal Activity of Monoterpenes and Related Compounds, Medicinal Chemistry Research, 25, 2171-2178 (2016)

Bajorath, J. y otros tres autores, Computational Methods for Medicinal Chemistry, Journal of Medicinal Chemistry 58 (3), 1019-1019 (2015)

Buckner, F.S. y J.A. Urbina, Recent developments in sterol 14-demethylase inhibitors for Chagas disease, International Journal for Parasitology, 2, 236-242 (2012)

Can, A., Quantitative structure-toxicity relationship (QSTR) studies on the organophosphate insecticides, Toxicology Letters, 230, 434-443 (2014)

Ensuncho, A., S. Figueredo y J. Robles, Modelado de las Relaciones Cuantitativas Estructura-Actividad (QSAR) de los Derivados 5-(Nitroheteroaril)-1,3,4-tiadiazol con Actividad Leishmanicida, Información Tecnológica, 28, 191-202 (2017)

Gascón, J. y otros catorces autores, Diagnosis, Management, and Treatment of Chronic Chagas' Heart Disease in Areas Where Trypanosoma cruzi Infection Is Not Endemic, Rev. Esp. Cardiol., 60, 285-293 (2007)

Guerra, C. y otros 4 autores, Análisis Qsar-2d De Los Derivados De 1,4-di-N-Oxidos de Quinoxalina con Actividad Contra la Enfermedad de Chagas, Química Nova, 39, 647-654 (2016)

James, M. y otros nueve autores, Second Generation Analogues of the Cancer Drug Clinical Candidate Tipifarnib for AntiChagas Disease Drug Discovery, J. Med. Chem, 53, 3887-3898 (2010)

Karp, J. y otros cinco autores, Current Status of Clinical Trials of Farnesyltrans-Ferase Inhibitors, Curr. Opin. Oncol., 13, 470-476 (2001)

Kraus, J.M. y otros 5 autores, Rational Modification of a Candidate Cancer Drug for Use Against Chagas Disease, Journal of Medicinal Chemistry, 52 (6), 1639-1647 (2009)

Kubinyi, H., 2D QSAR models: Hansch and Free-Wilson analyses, Comput. Med. Chem. Drug. Discov, 5, 539-570 (2004)

$\mathrm{Li}, \mathrm{J}$. y otros siete autores, QSAR Study on Estrogenic Activity of Structurally Diverse Compounds Using Generalized Regression Neural Network, Sci. China Ser B-Chem, 51, 677-683 (2008)

$\mathrm{Li}, \mathrm{Q}$. y otros diez autores, Benzimidazolones and Indoles as Non-Thiol Farnesyltransferase Inhibitors Based on Tipifarnib Scaffold: Synthesis and Activity, Bioor. Med. Chem. Lett. 15, 2918-2922 (2005)

López, J.M., A. Ensuncho y J.R. Robles, Diseño de Fármacos Anticancerosos Derivados de Cis-Platino(li) Mediante la Técnica QSAR, Basada en el Funcional de la Densidad, Inf. Tecnol., 25, 161-172 (2014)

Neese, F. The ORCA Program System, Wiley Interdiscip. Rev: Comput. Mol. Sci., 2, $73-78$ (2012)

Nikolic, K., S. Filipic y D. Agbaba, QSAR Study of Imidazoline Antihypertensive Drugs, Bioorganic \& Medicinal Chemistry, 16(15), 7134-7140 (2008)

Rassi, A. Jr., A. Rassi y J.A. Marin-Neto, Chagas Disease, The Lancet, 375, 1338-1402 (2010)

Roy, K. y otros cinco autores, Some Case Studies on Application of "rm2" Metrics for Judging Quality of Quantitative StructureActivity Relationship Predictions: Emphasis on Scaling of Response Data, J. Comput. Chem., 34 1071-1082 (2013)

Roy, K. y S. Kar, The rm2 Metrics and Regression through Origin Approach: Reliable and Useful Validation Tools for Predictive QSAR Models (Commentary on 'Is regression through origin useful in external validation of QSAR models?), Eur. J. Pharm. Sci., 62, 111-114 (2014)

Rueda, K. y otros tres autores, Transmisión oral de Trypanosoma cruzi: Una Nueva Situación Epidemiológica de la Enfermedad de Chagas en Colombia y otros Países Suramericanos; Biomédica, 34, 631- 641 (2013) 
Scior, T. y otros seis autores, How to Recognize and Workaround Pitfalls in QSAR Studies: A Critical Review, Current Medicinal Chemistry, 16, 4297-4313 (2009)

Tetko, I. y otros once autores, Virtual Computational Chemistry Laboratory - Design and Description, J. Comput. Aid. Mol. Des., 19, 453-63 (2005)

Tropsha, A., Best Practices for QSAR Model Development, Validation, and Explotation, Molecular Informatics 29(6-7), 476-488 (2010)

Tropsha, A., P. Gramatica y V.K. Gombar, The Importance of Being Earnest: Validation is the Absolute Essential for Successful Application and Interpretation of QSPR Models, QSAR \& Combinatorial Science, 22(1), $69-77$ (2003)

Valerio, L., M Sabria y A. Fabregat, Las Enfermedades Tropicales en el Mundo Occidental, Medicina Clínica, 118, 508514 (2002)

Veerasamy, R. y otros cinco autores, Validation of QSAR Models-Strategies and Importance, Int. J. of Drug Design and Discovery, 2(3), 511-519 (2011)

WHO, Chagas Diseases (American Trypanosomiasis), World Health Organization, Geneva, Switzerland (2018)

Xinliang, Y. y W. Xueye, Prediction of Glass Transition Temperatures of Aromatic Heterocyclic Polymers, International Journal of Materials Research, 2, 329-335 (2012)

Zhou, W. y otros cuatro autores, High-Dimensional Descriptor Selection and Computational QSAR Modeling for Antitumor Activity of ARC-111 Analogues Based on Support Vector Regression (SVR), International; Journal of Molecular Sciences, 13, 1161-1172 (2012) 